\title{
Data processing in microbiology: an integrated, simplified system
}

\author{
GL RIDGWAY, J BATCHELOR, * AUDREY LUTON, AND M BARNICOAT \\ From the Departments of Microbiology and *Major Computer Project, University College Hospital, \\ London WCIE 6AU, UK
}

SUMMARY A MUMPS based computer system is described for the processing of data in a microbiology laboratory. The system uses visual display units and mnemonic codes for data input. All functions are carried out within the department by the medical, technical, and clerical staff. While the system described is integrated with other user-systems in the hospital, it is readily adaptable, and portable to a stand-alone system.

Pathology laboratories are faced with an increasing workload at a time of increasing financial stringency. The acquisition of further personnel in the laboratory is being thwarted by budgetary restraints. In order to cope with both the greater complexity of the work and the increase in numbers of specimens, and yet keep revenue costs within acceptable limits, it is becoming increasingly necessary to look at computerassisted data processing.

A number of systems applicable to microbiology have been reported. Periodic analysis of paper tape coded data was described by Alexander et al., ${ }^{1}$ while the use of paper tape for reporting and analysis was reported by Mitchison et al. ${ }^{2}$ Punch card systems were described by Whitby and Blair, ${ }^{3}$ Goodwin and Smith, ${ }^{4}$ and Goodwin, ${ }^{5}$ the latter using the Portapunch system. In an effort to speed the transfer of data from the laboratory bench, two similar systems using optimal mark reading (OMR) have been reported. ${ }^{6} 7$

In the above systems, it appears that an extra step has been introduced into the laboratory work pattern, the burden of which falls on either the secretarial or technical staff. Microbiology reporting differs from that in biochemistry and haematology significantly in that most of the data destined for patients' notes are not in numerical form. This places a stringent requirement for flexibility on any computer system for microbiology reporting.

\section{Objectives of the present system}

The Microbiology Laboratory at University College Hospital, London, handles more than 140000

Received for publication 19 February 1980 specimens per year, with a current compound increase in the workload of approximately $12 \%$ per annum. Computer-assisted reporting was originally introduced using an OMR based system, linked to the hospital main-frame computer then in use, a Rank Xerox Sigma 6. Owing to an unacceptably high failure rate of both peripheral and central hardware, the system had to be prematurely abandoned. On return to a manual reporting system, it rapidly became apparent that computer processing was a necessity. The hospital main-frame was at about that time (1977) replaced with a PDP 11/70 running MUMPS software. We were thus presented with the challenge of designing a new system to process our workload using unfamiliar software against a background of suspicion from the laboratory staff.

The following laboratory objectives were required from the new system:

1 The workflow pattern of the laboratory should require minimal alteration from the manual-reporting system.

2 The computer should be available day and night, seven days a week.

3 The system should be reliable with minimal down-time.

4 The laboratory staff should not be held to specific times for reporting, checking, and printing reports on grounds of computer availability alone. As far as possible, all reports should be printed in the laboratory.

5 The system should be easy to use. Input should be simple and rapid. Extra work for medical, technical, and clerical staff should be minimal.

6 Coding should be simple to learn and preferably in mnemonic English. 
7 The system should minimise reporting errors without removing from the laboratory staff responsibility for the technical accuracy of their work.

8 Flexibility should be such that non-standard reports could be easily entered.

9 Rapid recall of current and previous results should be possible, with facility for accumulated results to be viewed if required.

10 Data for epidemiology, work load statistics, quality control, and infection control should be regularly produced by the computer without further data input.

\section{The hardware}

In October 1977, a DEC PDP 11/70 minicomputer running MUMPS (Massachusetts General Hospital Multi-User Programming System) software was installed to support a number of on-line patient administration and laboratory systems. The configuration now (November 1979) includes provision for up to 80 terminals (50 so far installed) and 350 million characters of disk storage. The present users apart from Microbiology, include the Patient Master Index, the Inpatient Bed-state, the Disease Index, Outpatient appointments, and Radiology.

The Microbiology Department is equipped with 10 Hazeltine 1500 visual display units (VDU) (9 in the main laboratory area, and 1 in a satellite laboratory in the Department of Genitourinary Medicine), running at 9600 baud for request and result entry/ amendment, checking, and enquiry. Two 300 baud printers produce identification labels, and an 1800 baud printer produces reports and various other listings. Other fast printers are obtainable elsewhere in the hospital as back-up for the single printer in the Microbiology Laboratory.

\section{Description of the system}

The workload conveniently divides into three categories. These are: (i) bacteriology cultures; (ii) virology and serology; and (iii) Department of Genitourinary Medicine (GUM). This latter department provides $30 \%$ of the Microbiology Department's total work, much of which is processed on site in the GUM Department, and that system will be described separately below.

\section{REQUEST ENTRY}

Specimens and requests are divided on arrival into category i or ii above. Requests may be entered at any VDU. Laboratory numbers are assigned sequentially by the computer in a range determined by whether the VDU is associated with category i or category ii work (1 to 30000 for i, and $>30000$ for ii). Numbering is re-started on the first day of each month, incorporating the number of the month and the year (thus a bacteriology culture specimen in November 1979 could be numbered 1483/11/79). Figure 1 shows the culture request form in use, a similar format being used for the serology and antibiotic request form. This form was originally of the NCR type, but the flimsy top copy and carbon impregnation are no longer used.

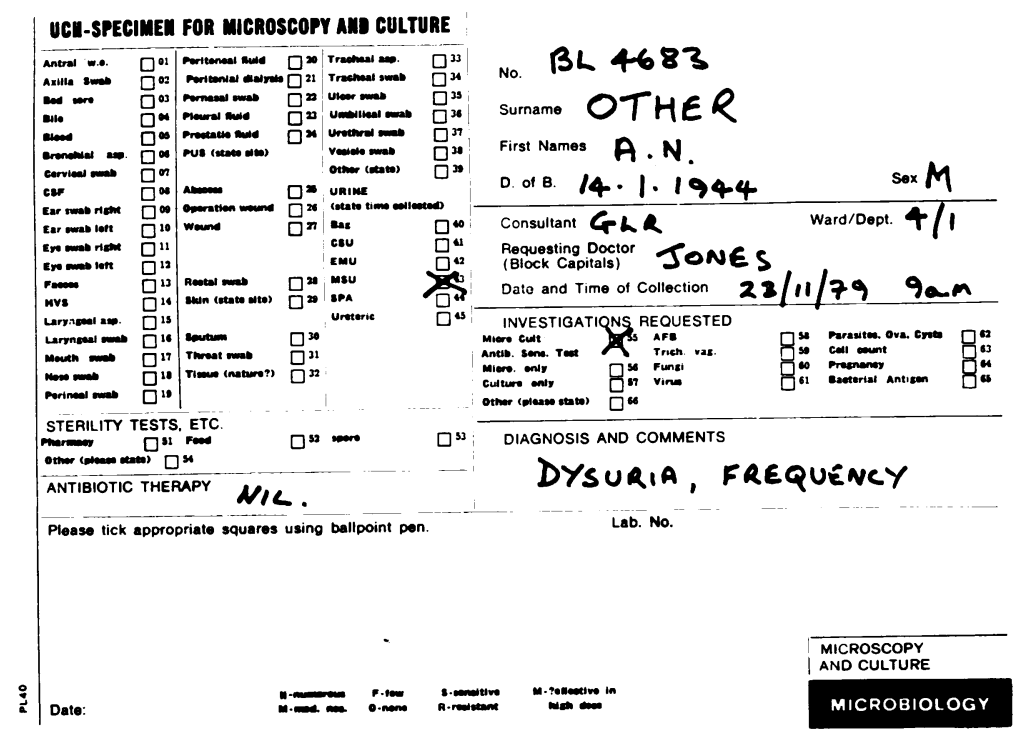

Fig. 1 Microbiology request form. 
If the patient has a hospital number, this is entered using the typewriter keyboard on the VDU. The link with the Patient Master Index results in the computer producing the details of a patient's name, etc. On verification, the computer then checks the bed state files and, if the patient is known, will produce details of ward and consultant for verification. When the above data are not known to the computer, the user is prompted to supply the data. In the absence of a hospital number, the computer assigns a ' $Z$ ' laboratory patient number, which may be accessed in the same way as a standard hospital number. However, the computer makes two attempts to match ' $Z$ ' numbers against other patient numbers to avoid possible duplication of records. The first is at the moment the new patient details have been entered. The second is a more sophisticated check, which is made overnight. A repeat facility allows the rapid entry of multiple requests from the same patient or from the same clinic.

Information on specimen type and tests required is entered using the numerical code shown on the request form (Fig. 1). Abbreviated details of antibiotic therapy and reason for request are also input. An associated printer produces two labels, one for the specimen and one for the request card. Each bears the laboratory number, name of the patient, and specimen and investigation type. Request details may at any time be redisplayed and amended if required.

\section{RESULT ENTRY}

The specimen is processed using the standard laboratory methods. All relevant working is recorded on the back of the request card. Negative results are returned to the secretarial staff for reporting, while positive results are reported by the technical staff. The laboratory number is input at a VDU. This will produce a shortened version of the request, allowing verification. The program allows for a series of result types, each of which has its own skeleton format. A result is entered by specifying the mnemonic code for the result type and the key information details, for example, an organism code, numeric titre, or free text comment, as appropriate. The computer then displays the expanded version of the result for verification. A report is composed of any combination of result types in any order. At all stages the computer will prompt for the required information but, as the laboratory staff become proficient in the use of the system, the prompts can be bypassed using spaces to separate the result codes. Where a coded item is required, and the code is unknown to the user, input of '?' will produce a list of the relevant codes. If at any time the user wishes to report non-standard data, free text can be inserted instead of a code.

It is often desirable to suppress certain results, for example, some antibiotics, or comments addressed to the medical staff of the laboratory. These are displayed within parentheses, and although they will be available for scrutiny on the laboratory VDUs, they will not appear on the final printed report or on VDU enquiries from outside the laboratory.

The repeat facility allows the rapid entry of multiple identical results, for example, negative urine screens.

\section{CHECKING RESULTS}

As required, designated staff are able to use the checking program to 'sign-out' reports from a VDU. The user is identified by a personal code and proceeds to check reported results. The computer presents the request form first, followed by the results. The checker confirms the results, or may alter them as required. Available on the screen is the status of the report (preliminary or final), the date and time of reporting, the initials of the member of staff reporting, and when or if any previous reports have been printed. If there is any question concerning the result, it may be held back for further checking against the work card. Otherwise the report is released for printing.

\section{PRINTING OF REPORTS}

The fast printer produces reports of the format shown in Figure 2. These are produced, ready sorted according to destination, and will bear the full name of the checker. The only restrictions on printing time are the internal and external delivery services.

\section{DEPARTMENT OF GU MEDICINE}

In this department worksheets are employed. The sheet is identified to the computer by a sheet number. Patient number and microscopy results are entered on the sheet and are entered at a VDU when the sheet is complete. The screen will show a replica of the worksheet, and the results are filled in. When culture results are known, the worksheet is recalled on the VDU, and the results are entered again in the worksheet format. Patient numbers and worksheet numbers are protected by a check digit. Serology requests and results are processed similarly. Individual printed results for patient are produced as above. Confidentiality is preserved by the use only of patients' numbers and the restriction of access to a patient's results.

\section{ENQUIRIES}

At any time laboratory staff may see the accumulated results for a patient; all available information will 


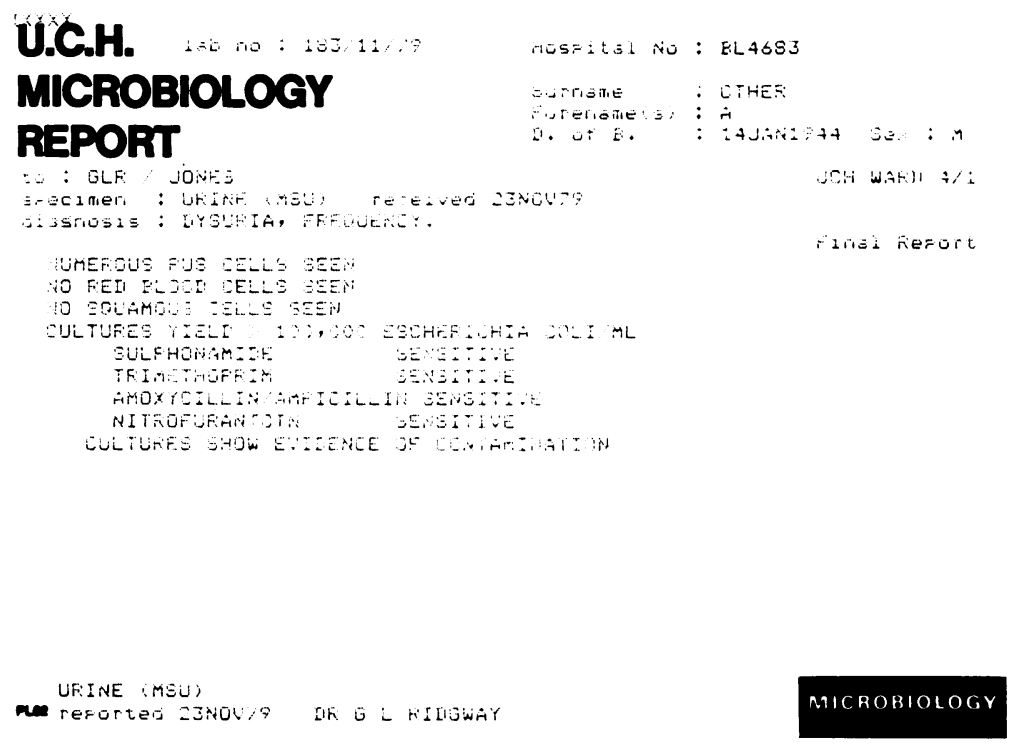

Fig. 2 Microbiology result format.

be displayed. This allows rapid response to telephone requests. If a result is not available, the VDU will give an indication of where the specimen may be found in the laboratory. If required, a printed copy of the accumulated results may be obtained from the printer. Outside the laboratory results which have been released are available to the clinician on VDUs.

\section{OTHER FACILITIES}

The computer produces overnight printouts of positive reports, weekly and monthly infection control data, a day book and work in progress list, an overdue request list, quality control and workload data, and a number of individual research listings. Other listings may be available as required. The system is being continually improved. New experimental programs include text processing, the monthly theatre infection report, a message box, a facility for ad hoc data searches, and the department's multiple-choice question bank.

\section{Advantages of using MUMPS}

MUMPS is a combination of programming language, file management system, and operating. ${ }^{8}$ The comprehensive nature of the software, together with the definition of an American National Standards Institute (ANSI) Standard $^{9}$ guarantees simple portability of systems written in MUMPS between different computers. MUMPS was developed in a hospital environment (at the Massachusetts General
Hospital) and therefore includes many features which are of particular advantage in developing pathology reporting systems, especially for microbiology.

TERMINAL HANDLING

MUMPS can support a large number of on-line terminals and provide very fast response times. It is straightforward to use timeouts to ensure that terminals left unused are logged off automatically for security purposes. Specific terminals can easily be recognised so that, for example, requests may be entered in more than one laboratory and the specimen labels automatically printed in the appropriate laboratory.

INTERPRETED LANGUAGE

This allows programs to be written rapidly and then amended quickly to take account of the lessons learnt during operation of the system.

VARIABLE LENGTHS

All data items are variable length character strings. Thus results may be entered either as short mnemonic codes or as long sentences without sacrificing efficient storage of data.

BINARY-TREE PHYSICAL FILE STRUCTURE This permits rapid direct access to a large data base so that all results may be stored in a single file which simplifies enquiries and permits access or amendment at any time. 
MULTILEVEL FILE STRUCTURE WITH

VARIABLE-LENGTH STRING SUBSCRIPTS

This permits a flexible system design which is geared to the user's needs rather than imposing restrictions through the operating system.

\section{Discussion}

The system described has proved highly effective. Breakdowns have proved to be relatively rare and minor events. The impact on the laboratory workflow pattern is thus minimal. Should major breakdown occur, a temporary return to manual reporting would not cause major disruption.

It was decided that all data should be input at asynchronous VDUs operating one prompt at a time. These are now sufficiently cheap that they could be installed in most of the locations where staff need to use them. Thus the work of the laboratory is not dependent on single, expensive peripherals such as OMR or, since all staff operate the system, on one or two critical members of staff. The VDUs are connected to the computer by different multiplexers and line driver racks. Thus reporting of results can continue despite failures in any one of these pieces of equipment and is interrupted only by the rare failure of the central computer and its disks. (These are backed up by a two-hour service contract.) Routine maintenance is arranged outside routine laboratory working time.

VDUs have the advantage that any program may be run at any terminal. Thus no reporting function is held up for lack of a specific terminal somewhere in the department. Whenever any laboratory number, patient number or code is keyed in the computer can always echo back corresponding details or expanded text so that the user can verify the accuracy of the items input. Since fixed format screens are not used it is possible for the user, when he cannot remember a code, to obtain a display of codes on the VDU screen. There is virtually no limit to the number of alternative codes possible under the system, and more can be added at any time, but if no suitable code is available the user always has an option to enter free text.

The use of structured program design techniques makes the transactions easy to follow, and various shortcut procedures speed up the entry of repetitive requests and results.

Because of the MUMPS file-sharing facility the details of any specimen are available on-line at any time at any VDU and may be accessed by laboratory number, patient number, or patient name.

Clerical workload has decreased, allowing more efficient use of the limited staff available. Patient data are available on-line 23.5 hours a day until three months have elapsed since the most recent request was received from a patient (TB and antenatal patients one year). After this time results are transferred to an archive file where they may be retained on-line as long as disk space permits. At regular intervals the complete database is copied to microfiche in patient name order to provide, at nominal cost, a permanent and compact hard copy of all work performed in the laboratory. (Six months' results may be stored on either side of a single leaf in an A4 binder.) The system does slightly increase technician time, but the benefits (in particular rapid recall of previous results) outweigh this increase. Checking of reports can be performed by one clinician in about $\mathbf{4 5}$ minutes per day. Initial training of each member of the laboratory in the use of the system consisted of three one-hour sessions conducted by the computer department taking groups of three at a time. All training of new staff has been performed internally by the laboratory staff. We have found that staff at all levels rapidly become proficient and that the arrangements remain popular.

We believe that the system described here is the first transferable computer system for microbiology reporting to be developed in the UK and that it would enable any large laboratory to provide, at a reasonable cost, a better service than would be possible using manual methods. It is hard to quantify costs, particularly revenue costs, since the microbiology system at UCH runs as part of a MUMPSbased hospital information service and accounts for only about a quarter of the total load on the computer. We believe this is the most advantageous way to provide computing in a hospital environment and also the most economical. However, the system can equally well run on a stand-alone basis, and other hospitals are interested in installing it on this basis. For those considering such a move the following facts should be relevant. The hardware to support a stand-alone system currently costs up to about $£ 40000$ with about $15 \mathrm{MB}$ of disk storage, depending on the workload of the laboratory. All data input is performed by existing laboratory staff without increasing work-load. The system was developed and installed with about 1.5 man years of effort from the UCH computer project. No further changes to the system are now envisaged other than the addition of new codes, which is under the control of the laboratory. The work involved in setting up the system for another site is minimal. One person with computer expertise would be required for about one year to arrange for installation of the computer, setting up the system, training of staff, and making any modifications required locally. One or two technical staff would need to be trained by this person to perform the 
file backups which take about 0.5 hours per day. (In the UCH computer centre, 1.5 operating staff are employed because there are three powerful computers located there, but the microbiology system takes a negligible amount of their time.) It would also be most helpful to have software support available on a regional basis.

Given the falling costs of computer equipment and the rising costs of staff salaries, many laboratories will need to consider computer-assisted reporting in the near future.

We gratefully acknowledge the co-operation and assistance of the staff of the Microbiology Laboratory, Major Computer Project, and the Department of Genito-urinary Medicine, UCH, London WC1, in the development and implementation of this system. Also, our thanks to Mr Peter Luton, of the Department of Microbiology, for providing the illustrations.

\section{References}

${ }^{1}$ Alexander MK, Connigale J, Johnson T, Poulter IR, Wakefield J. A data processing system for hospital bacteriology. J Clin Pathol 1970;23:77-80.
${ }^{2}$ Mitchison DA, Darrell JH, Mitchison R. A computerassisted bacteriology reporting and information system. $J$ Clin Pathol 1978;31:673-80.

${ }^{3}$ Whitby JL, Blair JN. Data processing in hospital bacteriology: experience of 18 months trial. J Clin Pathol 1972; 25:338-43.

- Goodwin CS, Smith BC. Computer printing and filing of microbiology reports. 1-Description of the system. $J$ Clin Pathol 1976;29:543-52.

${ }^{5}$ Goodwin CS. Computer printing and filing of microbiology reports. 2-Evaluation and comparison with a manual system, and comparison of two manual systems. J Clin Pathol 1976;29:553-60.

- Andrews HJ, Vickers M. An assessment of one year of computer-assisted microbiology reporting at Charing Cross Hospital. J Clin Pathol 1974;27:187-91.

7 Williams KN, Davidson JMF, Lynn R, Rice E, Phillips I. A computer system for clinical microbiology. J Clin Pathol 1978;31:1193-201.

${ }^{8}$ Bowie J, Barnett GO. MUMPS-An economical and efficient time-sharing system for information management. Computer Progress in Biomedicine 1976;6:11-22.

- Neil JT, ed. MUMPS Language Standard. NBS Handbook No. 118. Washington DC: US Govt Printing Office, 1976.

Requests for reprints to: Dr GL Ridgway, Department of Clinical Pathology, University College Hospital, Gower Street, London WC1E 6AU, UK. 